\title{
Anabases
}

ANABASES Traditions et réceptions de l'Antiquité

\section{Annelies Lannoy, Corinne Bonnet et Danny Praet (éds.), " Mon cher Mithra ». La correspondance entre Franz Cumont et Alfred Loisy}

\section{Philippe Foro}

\section{(2) OpenEdition \\ Journals}

Édition électronique

URL : https://journals.openedition.org/anabases/13188

DOI : 10.4000/anabases.13188

ISSN : 2256-9421

Éditeur

E.R.A.S.M.E.

\section{Édition imprimée}

Date de publication : 29 octobre 2021

Pagination : 289-290

ISSN : $1774-4296$

\section{Référence électronique}

Philippe Foro, « Annelies Lannoy, Corinne Bonnet et Danny Praet (éds.), « Mon cher Mithra ». La correspondance entre Franz Cumont et Alfred Loisy», Anabases [En ligne], 34 | 2021, mis en ligne le 29 octobre 2021, consulté le 05 décembre 2022. URL : http://journals.openedition.org/anabases/13188 DOI : https://doi.org/10.4000/anabases.13188 
Sophie Basch, Souvenir des Dardanelles. Les céramiques de Çanakkale, des fouilles de Schliemann aujaponisme, Bruxelles, Académie royale de Belgique, 2020, 137 p. / ISBN 978280310742I, I5 €.

Le voyage auquel nous invite Sophie Basch commence en Turquie ; la petite ville de Çanakkale, dans le détroit des Dardanelles, est connue, depuis le $\mathrm{xvIII}^{\mathrm{e}}$ siècle, pour sa production de céramiques destinées au marché local, mais aussi à l'exportation. “Lointaines descendantes des idoles anatoliennes primitives, du bestiaire assyrien, des céramiques seldjoukides et de l'orientalisme hellénistique» (p. II), ces artefacts constituent un cas bien singulier de réception de l'Antiquité. En effet, lancée sur leurs traces, Sophie Basch croise Schliemann, Flaubert, Gauguin et même Picasso! En onze brefs chapitres, écrits d'une plume alerte qui embarque le lecteur, Sophie Basch parcourt les méandres d'un voyage, entre Turquie et Japon, céramique et peinture, Homère et les Demoiselles d'Avignon. On part donc de l'histoire connectée de la céramique et du succès des poteries des Dardanelles: icones d'une région, voire d'une identité (la turcité) d'un style et d'une époque, vendues, échangées, photographies, commentées, collectionnées, elles suscitent une passion peu banale. Devenue un " lieu de mémoire », elles n'en sont pas moins l'objet d'appropriations multiples, y compris en Grèce, suscitant alors, de part et d'autre, des discours embarrassés qui visent à nationaliser les productions de Çanakkale, quitte à convoquer le passé loin- tain, les traditions populaires, les potiers homériques ou ceux de Troie! Le face à face entre Ottomans et Grecs ne suffit cependant pas à rendre compte du destin international des pittoresques céramiques de l'Hellespont. Voyageurs, explorateurs, scientifiques d'Europe et d'Amérique, férus de curiosités locales et de porcelaine, s'emparent du sujet et comparent avec ce qu'ils connaissent, comme la faïence de Delft ou celle du Japon. La poterie de Çanakkale fait alors son entrée au musée; elle intéresse, mais suscite aussi un certain dégoût car grossière, voire hideuse, mais fascinante. Mais les goûts évoluent et ce qui semblait kitsch hier devient élégant; Flaubert, en I85I, qualifie de “ fantastiques » les monstres qui les ornent, tandis que Nerval n'hésite pas à parler de chef-d'œuvre. N'y a-t-il pas quelque souvenir du cheval de Troie capturé dans ces objets décorés d'animaux? Pendant ce temps, les expositions universelles de la fin du xIx ${ }^{\mathrm{e}}$ siècle acclimatent l'œil occidental à la céramique, à la faïence, des objets traditionnels qui semblent exprimer une résistance à l'industrialisation et même conserver un savoir-faire qui remonte à l'Antiquité. Prétendument immuable, le travail de la céramique fait figure d'antidote à la modernité galopante. Face à l'Occident triomphant, l'Orient joue la carte de la tradition, comme en témoigne le japonisme. Ni Mallarmé ni Proust ne restèrent indifférents à cet engouement pour la glaise, tandis qu'en i87o, le renouveau national passe par un retour à la nature, y compris dans les arts. Sophie Basch revient sur l'exposition universelle de i878 et l'accueil enthousiaste 
réservé aux arts du Japon : c'est une véritable révélation, dont bénéficient collatéralement les poteries des Dardanelles, perçues comme l'expression vivante d'un art millénaire qui plonge ses racines dans les strates d'Hissarlik. Schliemann lui-même n'hésite pas à jeter un pont entre les céramiques des boutiques de l'Hellespont et les débris dégagés dans ses fouilles de Troie. De la chouette d'Athéna aux vases zoocéphales “ post-homériques » de Çanakkale, il n'y avait qu'un pas. L'ouvrage se termine sur d'étranges Demoiselles d'Avignon, qui ne sont pas celles que l'on croit. L'Épilogue du livre retisse brillamment les fils d'un itinéraire aussi étonnant qu'amusant et instructif. Tissée de malentendus, transferts, rapprochements, coups de cœur et poussées nationalistes, cette histoire qui fourmille d'acteurs (potiers, artistes, savants, marchands d'art, photographes, écrivains, hommes politiques, critiques d'art, responsables de musée, journalistes, voyageurs, diplomates...) éclaire singulièrement l'histoire du goût, qui est bien une histoire connectée, "par ricochet », dans le temps long qui va de l'Antiquité au $\mathrm{xx}^{\mathrm{e}}$ siècle.

Corinne Bonnet

Université Toulouse - Jean Jaurès corinne.bonnet@univ-tlse2.fr

Jonas Borsch, Olivier Gengler, et Mischa Meier (éds.), Die Weltchronik des Johannes Malalas im Kontext spätantiker Memorialkultur, Stuttgart, Franz Steiner Verlag, 20I9, 372 p. / ISBN 97835ı5ı20III, $64 €$.

Like clockwork the Project Malalas, located at the University of Tübingen and sponsored by the Heidelberg Academy of Sciences and Humanities, keeps churning out new volumes. Since its inception in 2014 the project group, led by Mischa Meier, has published three entries to the series "Malalas Studien", with two more already in production. The present review is concerned with the third installment, based on a conference held in Tübingen in the autumn of 20r6, entitled "Die Weltchronik des Johannes Malalas im Kontext spätantiker Memorialkultur". In contrast to the first two volumes, that were focused on Malalas, the textual genesis of the chronicle and its source material, this book deviates from the author centric approach and instead paints a panoramic view of memorial culture in Late Antiquity. As the editors, Jonas Borsch and Olivier Gengler, explain in their introduction, this methodological shift is deemed necessary, because Malalas himself does not give us any explicit information on his view of the past. Therefore, they decided to take a detour and embed the chronicle as broadly as possible within the ancient memorial landscape to better comprehend (and expose) Malalas' underlining historical conception as well as the resulting selection criteria of the Chronographia.

The following "detour" consists of i3 contributions, further divided into six subsections: the rubric "Historiographie als memoria" is comprised of Karl-Joachim Hölkeskamp's study on the self-fashioning of the Republican gens Fabia. Three studies are dedicated to the theme of "Memoria und Kaisertum". Jonas Borsch analyzes the over Ioo physical descriptions of important individuals (mainly emperors and heroes, but also the apostles Peter and Paul) integrated into the chronicle. He argues that these "written portraits" were not simply copied from other sources but rather reflect Malalas' attempt to create historical continuity through visual and topographical anchoring. The portraits thus form an ancestral gallery of Roman history. Laura Mecella then scrutinizes the account of Trajan's Parthian campaign with an emphasis on the fictitious occupation of Antioch. She traces Malalas' invention of this event to an Antiochene source 
that combined three different strains of local memoria. Hanns Christof Brennecke sketches the divergent paths of the Byzantine historio- and hagiographical tradition with a study of the (rare) positive image of the emperor Zeno displayed in the Life of Daniel the Stylite.

The next two contributions are concerned with the "Ausformungen kirchlicher memoria". Volker Menze looks at the reception of the Council of Chalcedon. Following Pierre Nora's conception of lieux de mémoire, he argues that even one hundred years after its convocation the council remained a mémoire vivante that was continuously altered and (re-)interpretated by its proponents and adversaries alike. Therefore, Malalas only briefly discussed Chalcedon and refrained from including a clear doctrinal position in his chronicle. Sebastian Watta probes how material culture, specifically the mosaic floors of Near Eastern churches, could impact and influence individual and collective memory. Fueled by the strife for salvation, the mosaics were part of a complex system of sacralization of and within the churches that combined permanent visual stimuli with (frequent) liturgical acts to create a continuous "cultural memory".

The following section is concerned with "Die Stadt als Erinnerungsträger". Emmanuèle Caire first undertakes a caste study of Antioch's Epiphania area to demonstrate that despite the frequent mention of seemingly specific monuments Malalas' chronicle neither was an architectural treatise nor a topographical guide. According to Caire, the names of these monuments were more important than their actual nature or present condition. Even after they had ceased to exist, they could still serve as a mental marker for contemporary readers.

Thereafter, Philipp Niewöhner demonstrates that the preservation of the ancient cityscape of Milet throughout the early Byzantine period was intentional and should be viewed as an example of antiquarianism. This behavior was unrelated to paganism, but instead inspired by the numerous monuments themselves, which might also explain why Asia Minor developed no Byzantine architectural style of its own.

The rubric "Memoria unter Justinian" consists of two entries: Raf Praet contextualizes the Chronographia within the intellectual milieu of sixth-century Constantinople. He proposes a direct social connection between Malalas, John the Lydian and Cassiodorus that is not simply based on their common bureaucratic experiences and erudite networks, but also palpable in their treatment of Roman history. Praet tries to exemplify his hypothesis through a case study of their respective treatment of the color purple. Olivier Gengler then discusses the historical references embedded into numerous Novellae of Justinian, which he interprets as a conscious strategy to build and fortify a continuous Roman imperial memoria.

The last section "Die Chronik als Memorialgattung" starts off with an overview of the Latin chronicles of the fifth and sixth century by Carlo Scardino. Despite the conventions of the genre, Scardino clearly shows that each chronicler had ample opportunities to individualize and interpret the historical material. Christian Gastgeber discusses - and almost discards - the idea of a memoria of pagan history in the Chronicon Paschale. The interest in chronology trumped the notion of preserving pagan culture and ultimately drove the selection process of the author. Erika Juhász echoes this sentiment in her study of the Christian martyrs included in the Easter chronicle. The author seldomly strayed from the beaten paths of his main sources (in this case Eusebius and Malalas) and did so only to optimize his chronology, not for the sake of the glorification of the martyrs. 
The overall value of this entry to the "Malalas-Studien" derives from the interdisciplinary approach and wide range of topics covered under the umbrella of ancient memoria. The quality of the individual contributions is high as well, but for those strictly interested in Malalascentric scholarship this volume offers only four new additions to the field and therefore acts more as a prelude to a forthcoming volume on Malalas as a contemporary historian.

Christian Barthel Universität Potsdam christian.barthel@uni-potsdam.de

\begin{abstract}
Wim Broekaert, Elena Köstner et Christian Rollinger (éds.), The Ties that Bind. Ancient Politics and Network Research. Journal of historical network research, 4, Luxembourg, Université du Luxembourg, 2020, 356 p. / ISSN 25358863. En ligne uniquement : https:// doi.org/I0.255I/jhnr.v4io
\end{abstract}

Levolume édité par Wim Broekaert, Elena Köstner et Christian Rollinger est structuré en dix chapitres qui correspondent chacun à la contribution d'un auteur, laquelle offre une étude à part entière qui peut être considérée indépendamment des autres contributions. Chacune d'entre elles propose une mise en application des outils et méthodes tirés de la “Social Network Analysis » (SNA) sur un sujet d'histoire ancienne. La technicité déployée par les auteurs rend parfois l'ouvrage difficile d'accès pour un non-spécialiste des réseaux et l'on apprécie d'autant plus les clefs de lecture livrées dans le premier chapitre - sous forme de prologue - rédigé par Christian Rollinger.

Bien que les différentes contributions couvrent à la fois des éléments d'histoire grecque et romaine, l'accent est volon- tairement mis sur cette dernière, en particuliersurlafin del'époque républicaine et le début de l'Empire. La difficulté d'obtenir des ensembles de sources adaptés à l'analyse de réseaux - possibilité d'identifier un nombre suffisant d'acteurs et leurs relations - entraine une certaine redondance dans le choix des corpus étudiés. Ainsi, l'important corpus de lettres produit et reçu par Cicéron est examiné par Christian Vogel au chapitre 4, mais aussi par Christina Rosillo-López au chapitre 5 et Gregory Gilles au chapitre 6. Les auteurs évitent cependant les répétitions en proposant chacun un axe méthodologique qui leur est propre. Il s'agit là de l'objectif principal de l'ouvrage: illustrer un inventaire de méthodes dérivé de la SNA et adapté au traitement des sources anciennes par sa mise en application. L'ambition des auteurs n'est donc pas de remettre en cause des conclusions historiques bien établies par ailleurs, mais plutôt d'évaluer dans quelle mesure un traitement des relations interpersonnelles en analyse de réseaux permet de nuancer certains aspects relatifs à ces mêmes conclusions.

Ainsi, le chapitre 2, "Athens as a Small World ", réalisé par Diane Cline, s'insère dans la continuité des travaux déjà menés dans le courant des années I99o par Philip Stadler et Anthony Podlecki qui se sont intéressés à l'influence de l'ego-réseau de Périclès dans le conflit qui l'oppose à Cimon dans l'Athènes du $v^{\mathrm{e}}$ siècle av. n.è. À partir d'un large spectre de documents extraits principalement des œuvres de Plutarque et de Platon, Diane Cline restitue et caractérise un réseau de plus de sept cents relations dans lequel sont évaluées les positions relatives de Nicias, Cimon, Alcibiade et Périclès. Sur la base de critères formels et quantitatifs, elle démontre avec rigueur que le réseau athénien du milieu du $\mathrm{V}^{\mathrm{e}}$ siècle présente les propriétés structurales d'un "Small World » : chaque acteur peut atteindre n'importe quel autre acteur du 
réseau en passant par un nombre minime d'intermédiaires.

Dans la contribution suivante, “Quintus Cicero and Roman rule-Networks between centre et periphery ", Christian Vogel examine l'ego-réseau de Quintus Tullius Cicero, le frère cadet de l'orateur Cicéron. L'analyse se focalise sur l'exploitation des relations informelles par Cicero (les amicitiae) comme modalité de contrôle des territoires dans les régions périphériques de l'Empire. En mobilisant la SNA, l'auteur montre que, dans le cas de l'Asie comme de la Gaule, la construction de relations hors du cadre institutionnel entre les dépositaires de l'autorité romaine et les élites locales, mais également entre membres de l'aristocratie, constitue un levier essentiel du système de domination déployé par Rome.

Dans une optique différente, l'étude de Christina Rosillo-Lopez, « Informal political communication and network theory in the Late Roman Republic ", se concentre sur l'identification des chaînes de circulation de l'information dans les conversations privées (sermones) entre membres de l'élite sénatoriale. L'auteure envisage deux cas de figures: celui de Cicéron qui occupe une position centrale au croisement de plusieurs chaînes d'informations, et celui de Marcus Caelius Rufus, un jeune sénateur romain. En explorant plus avant le cas de Cicéron, elle montre sa déconnexion progressive des cercles de discussions les plus influents à la veille du conflit entre César et Pompée. Par ce biais, l'étude justifie partiellement le ralliement de Cicéron à la cause pompéenne et met en lumière les risques encourus par les difficultés d'accès à l'information.

Sur une période équivalente, l'étude de Gregory Gilles, “The political, social and familial networks discerned from Cicero's Letters during the Civil War of 49-47 BC », revient sur la question des critères à la base du choix de ralliement des membres du sénat pour le parti de César ou de Pompée. En examinant en détail les interactions au sein du réseau de Cicéron et de M. Caelius Rufus, Gregory Gilles montre que l'ouverture des hostilités entre César et Pompée ne semble pas avoir d'impact significatif sur la distribution des connexions de leurs partisans: les relations qui précèdent le conflit tendent à se maintenir, y compris entre membres de camps opposés. Partant de ces connexions préexistantes, Gregory Gilles s'intéresse au poids des liens familiaux et de l'appartenance aux factions politiques sur le choix du camp de ralliement.

Wim Broekart, dans son chapitre " The Pompeian connection. A social network approach to elits and sub-elits in the bay of Naples », met en lumière la manière dont les interactions entre deux groupes de l'élite pompéienne hiérarchiquement liés entre eux (élites et “sous-élites ») structurent les politiques locales. L'approche retrace l'évolution du réseau de l'élite pompéienne sur une longue période, depuis sa conquête lors de la guerre marsique $-c .89$ av. n. è. - jusqu'à la période flavienne. L'étude mobilise en particulier les mesures du type centre-périphérie pour caractériser la position relative des membres de l'élite, ainsi que les mesures de connectivité qui permettent de délimiter les groupes familiaux dominants. L'arrivée et l'intégration de nouvelles familles dans le cercle politique de Pompéi sont également passées au crible de l'analyse de réseaux.

La contribution d'Elena Köstner, "Genesis and Collapse of a Network: The Rise and Fall of Lucius Aelius Seianus ", s'intéresse à l'évolution du réseau de relations du proche conseiller de l'empereur Tiberius. L. Aelius Seianus développe au fil des années un réseau à la fois dense et complexe de relations politiques qui atteint son apogée au cours de l'année 3i de n. è. La même année, le conseiller est accusé de complot contre l'empereur puis exécuté. La disparition de Seianus va bien sûr de pair avec la désintégration de son ego-réseau mais occasionne aussi une exposition 
renforcée au risque de condamnation pour ses anciens contacts. En examinant les relations des proches du conseiller condamnés ou relaxés, Elena Köstner montre que les décisions de justice sont moins dépendantes du lien avec Seianus que de la qualité des relations avec les membres de la famille impériale.

L'apport de Fabian Germerodt, “ Networking in the early Roman empire : Pliny the younger " s'articule autour de trois études de cas tirées du réseau de Pline le Jeune. L'objectif du chapitre est d'illustrer les rouages de la vie sociale et politique des élites romaines de la fin du $\mathrm{I}^{\mathrm{er}}$ au début $\mathrm{du}$ $\mathrm{II}^{\mathrm{e}}$ siècle. L'accent est d'abord mis sur les mécanismes d'entraide et d'obligation qui poussent Pline le Jeune à soutenir son jeune protégé, Gnaeus Pedanius Fuscus Salinator, dans son projet de mariage avec Iulia Paulina, fille de la sœur de l'empereur Hadrien; puis à prendre publiquement la défense de Corellia Hispulla, la fille de Q. Corellius Rufus, un ami de longue date, alors même que cet engagement l'expose aux attaques du futur consul, Gaius Caecilius Strabo. L'auteur explore plus largement la manière dont les réseaux de relations informelles peuvent être manipulés pour faciliter l'accès à une position avantageuse et maintenir la défense de ses intérêts.

Au chapitre 9, “ Network Management in Ostrogothic Italy. Theodoric the Great and the Refusal of Sectarian Conflict ", Christian Nitschke se penche sur les modalités de gestion mises en œuvre par Théodoric pour organiser et exploiter un vaste réseau d'acteurs dans la société ostrogothique de l'Italie du début du vi ${ }^{\mathrm{e}}$ siècle. Il s'intéresse plus spécifiquement au parcours d'Anicus Manlius Severinus Boethius, un noble romain au service des Ostrogoths. Au terme d'une brillante carrière politique qui le conduit à exercer la haute charge de magister officiorum, Boethius est finalement inculpé pour haute trahison, emprisonné puis exécuté. En raison du manque de sources, les causes précises de la soudaine disgrâce de Boethius restent à ce jour inexpliquées. En mobilisant l'analyse de réseaux pour réévaluer la documentation disponible, Christian Nitschke propose d'expliquer les motivations qui conduisent Théodoric à interrompre brutalement la carrière de son chef de chancellerie comme une volonté de réagencer les propriétés structurales de son propre réseau.

Enfin, la contribution de Johannes Preiser-Kapeller, "The ties that do not bind - Group formation, polarisation, and conflict within networks of political elites in the medieval Roman empire", étudie l'apparition des conflits au sein de l'élite byzantine $\mathrm{du} \mathrm{IX}^{\mathrm{e}}$ au $\mathrm{xIV}^{\mathrm{e}}$ siècle, en se concentrant notamment sur la famille des Skléros. En comparant les propriétés structurales d'autres réseaux similaires, comme celui des élites de la Chine Tang, Johannes Preiser-Kappeller cherche à expliquer les phénomènes de polarisation et d'émergence des conflits dans les groupes dominants sur la base d'éléments structuraux. Il mobilise notamment les mesures d'assortivité introduites par M. E. J. Newman qui sont un indicateur du degré de polarisation des réseaux.

Cet ouvrage marque ainsi un avancement important dans la mise en application des méthodes tirées de la SNA en histoire ancienne. Outre le fait qu'il s'agit du premier volume exclusivement consacré au sujet, les différentes contributions atteignent l'objectif fixé par les éditeurs : évaluer la valeur heuristique des outils extraits de l'analyse de réseaux tout en cernant les limites de ce type d'approche.

\footnotetext{
Clément Dutrey

Université Toulouse - Jean Jaurès clement.dutrey@outlook.com
} 
Maarten De Pource, Nathalie de HaAn et David Russer (éds.), Framing Classical Reception Studies. Different Perspectives on a Developing Field, Leyde, Brill, 2020, 298 p. / ISBN 97890044270I3, пI8 €

Est-il encore possible, en 2020, de chercher à "cadrer" les recherches sur la réception de l'Antiquité, compte tenu des nombreuses publications d'ouvrages ou d'articles sur la question qui se sont succédé ces dix dernières années, notamment chez les maisons d'éditions de tradition anglo-saxonne?

Sans reprocher à M. De Pourcq, N. de Haan et D. Rijser leur décision de publier le résultat de cette manifestation scientifique, le calendrier de publication ne joue pas en faveur d'une originalité dans l'environnement de la recherche. En effet, il regroupe treize articles, répartis en deux parties, issus d'un colloque tenu en 2013 à l'Université Radboud de Nimègue au Pays-Bas. Cependant, l'ouvrage présenté ici mérite d'être défendu, d'abord pour sa présentation des différentes méthodes utilisées pour « développer un champ de recherches ». Ensuite parce que les propositions faites dans l'ouvrage sont intéressantes et portent de réels questionnements importants, comme l'avancent les éditeurs dans leur introduction, en revenant sur les recherches novatrices de Charles Martindale et Lorna Hardwick; d'ailleurs cette dernière participe à la réflexion dans la première partie de l'ouvrage, on y reconnait aisément la démarche qui l'anime depuis le début des années 200o. Son article illustre parfaitement les relations entre l'Antiquité et les “ textes » ultérieurs, insistant sur la nécessité de faire dialoguer scientifiques, créateurs et le public qui « reçoit ». Clare L. E. Foster, ensuite, relève la fausse opposition impliquant lestermes méthodologiques de “Tradition » et de “ Réception », en prenant comme point de départ les réflexions de Charles Martindale, et les approches inhérentes aux différentes méthodolo- gies (comparatiste, mémoire culturelle, etc). Elle s'appuie aussi sur la notion de type/typologie, encore trop peu utilisée. Le troisième article de cette première partie, rédigé par Fran Middleton, s'intéresse à la question de la réception littéraire dans les manuscrits médiévaux dans sa dimension matérielle en prenant l'exemple d'une transcription byzantine de l'Iliade au $\mathrm{x}^{\mathrm{e}}$ siècle, en faisant appel à l'analyse des paratextes existants. Il s'agit de montrer comment le cadrage de la réception s'effectue de manière littérale. Dans un autre registre, et pour conclure cette première partie, Edith Hall et Henry Stead présentent leur projet de recherche "Classics and Class in Britain I789-Ig39 ", réflexion sur la réception de l'Antiquité au prisme de la construction de la structure de classe dans cet espace et durant la période définie. Si la présentation de la réception culturelle antique peut paraître parfois réductrice (Ancient Rome received ancient Greece, and ancient Greece received elements in its culture from all of its ancient neighbors, especially those in western Asia and North Africa), elle permet néanmoins de questionner les cadres méthodologiques d'une réception des textes anciens en Angleterre, entre le xvıI ${ }^{\mathrm{e}}$ et le $\mathrm{xx}^{\mathrm{e}}$ siècle.

La deuxième partie de l'ouvrage s'ouvre sur le mythe de Pyrame et Thisbé utilisé dans les prêches chrétiens au cours du Moyen Âge. Pietro Delcorno définit d'abord ce médium en y intégrant la notion d'allélopoièse. Dans les exemples présentés, le mythe ancien devient une lecture allégorique et morale, notamment lors des sermons du dimanche de la Passion. L'auteur démontre ici la manière dont les prêcheurs distordent un mythe populaire en leur temps pour le faire correspondre à leur discours auprès d'un public connaisseur ou non du mythe et d'Ovide, et illustre la façon dont la chaîne de réception entrecroise l'écrit, l'oral et parfois aussi l'image, lorsque les prélats font référence à des sculptures religieuses ayant le même sujet. 
L'article suivant, de Piet Gerbrandy, introduit l'anthropologie dans l'étude de réception en prenant pour cas d'étude le poème De reditu suo de Rutilius. Le chercheur applique la notion du " rite de passage " à ce récit du v viècle, racontant le désir du Romain de revenir dans sa famille en Gaule, sous domination gothique. Il démontre ainsi que le poème ne relève pas tant d'un voyage réel que d'un récit autobiographique symbolique grâce à l'utilisation de canons littéraires antérieurs.

Par la suite, Cecilia Pavarani prend pour exemple la nécrologie de Comenius par Leibniz, au $\mathrm{xvII}^{\mathrm{e}}$ siècle, en explicitant la manière dont le philosophe allemand rend hommage à son maître, dans son éloge, tant par le contenu que la forme utilisée. L’angle d'étude choisi est celui de la spécialité des deux savants allemands, la science de l'éducation moderne, Comenius étant considéré comme l'inventeur de la pédagogie moderne. Le recours à Virgile et Lucrèce sert, selon l'autrice, à affirmer l'universalisme de la pédagogie prônée par Comenius, entre Humanisme et Réforme.

Dans l'article suivant, Jeroen Jansen présente la publication du rhétoricien Jacob Duym en i6o6 de six pièces de théâtre, dont le but est de démontrer l'importance de la révolte des Néerlandais face à l'Espagne, de la nécessité de poursuivre le combat contre le Duc d'Alba. Pour ce faire, Jacob Duym utilise le mythe de Persée et Andromède, en guise d'avertissement et d'appel aux armes auprès de la population néerlandaise. Sa démarche s'appuie sur l'emphase auprès des lecteurs, en mélangeant allusions historiques, concepts et symboles culturels, afin “ d'activer un schéma mental de savoirs ».

Dans un but assez similaire, Ronny Kaiser étudie les publications humanistes (entre le $\mathrm{xv}^{\mathrm{e}}$ et le $\mathrm{xvII}{ }^{\mathrm{e}}$ siècle), tant sur le fond (le texte en lui-même) que sur la forme (le para-texte qui vient avec le texte notamment), en prenant pour exemples les biographies de Cornélius Nepos et Suétone.
Ici aussi, l'auteur montre l'importance de l'allélopoièse, de l'interaction étroite entre biographie et historiographie de ces œuvres, depuis leur origine jusqu'à leur réappropriation à l'époque moderne, alors que ces deux approches étaient déjà différenciées dans l'Antiquité.

La mise en scène du patrimoine antique dans les textes comme cadre rhétorique est au centre de la réflexion de Susanna de Beer, dans son article sur l'utilisation de la ville de Rome chez des poètes italiens des $\mathrm{xv}^{\mathrm{e}}$ et $\mathrm{xvI}^{\mathrm{e}}$ siècles. L'autrice fait appel notamment au concept de Pierre Nora, les lieux de mémoire, pour mieux comprendre pourquoi certains poètes, comme le Florentin Landino et le Romain Vitalis, convoquent l'image des ruines de la Ville Éternelle, soit pour la mettre en miroir avec une autre puissance contemporaine comme Florence, soit pour justifier un héritage religieux papal. C'est donc les domaines de l'identité et de la légitimité politique dont il est question. Les lecteurs découvrent un texte comme un touriste visite un site patrimonial alors que, paradoxalement, la majorité de ces poètes n'ont jamais vu le Colisée ou le Capitole romain : ils font donc appel à une mémoire collective.

Les deux derniers articles nous transportent dans le monde contemporain, d'abord celui du Brésil du $\mathrm{xx}^{\mathrm{e}}$ siècle, puis dans celui de la création audiovisuelle du début du $\mathrm{xxI}^{\mathrm{e}}$ siècle. Rodrigo Tadeu Gonçalves et Guilherme Gontijo Flores, tout d'abord, s'intéressent aux traductions brésiliennes très récentes des textes anciens, souvent influencées par l'École française, à partir de la poétique de Manuel Odorico Mendes, Carlos Alberto Nunes et Haroldo de Campos. Leur démonstration apporte un éclairage assez intéressant sur la façon dont une traduction remodèle complètement un texte ancien au prisme de la culture native de ces poètes. Dans le dernier article (et le moins convaincant de tous), Koen Vacano cherche à démonter l'intérêt d'une comparaison dépassant les frontières de l'Histoire et des cultures, 
entre Médée et la série américaine Breaking Bad. L'idée de base est séduisante mais elle est parcourue de scories méthodologiques ennuyeuses, dont celle de trouver absolument des liens inconscients entre thèmes antiques et œuvres contemporaines non historiques. Ainsi, pour l'auteur, la traduction du titre de Breaking Bad (“tourner mal») et la résolution de l'histoire du personnage principal trouve une comparaison idoine à celle de Médée, par l'intermédiaire du tertium comparationis et de l'harmatia. Dans ce cas, l'auteur conclut que, comme Walter White, Médée breaks bad.

Il revient enfin à David Rijser de conclure par un épilogue très intéressant, dont la base est similaire à celle de Koen Vacano, avec comme fil rouge la série Top of the Lake de la néo-zélandaise Jane Campion et les liens philosophiques avec l'Edipe de Sophocle. Mais la véritable réflexion est celle de la question de la réception, constituant un acte réfléchi et engagé : The past, in other words, is being subjected to a judgment excluvively based on the present. Chacun des articles l'a démontré, tant dans les méthodes de cadrage de l'Antiquité, dans leurs aspects théoriques, que dans les exemples développés, depuis l'Antiquité tardive.

En résumé, il faut redire l'excellente impression que nous laisse la lecture de cet ouvrage, dans lequel les articles sont accessibles et peu jargonneux. Les quelques images en couleur sont les bienvenues et les exemples développés au fil des chapitres sont assez originaux comptetenu des publications antécédentes sur le même sujet. Les “ cadres » analytiques très riches et les enjeux posés par les éditeurs sont largement respectés, et de manière convaincante.

Mathieu Scapin Musée Saint-Raymond, musée d'Archéologie de Toulouse mathieu.scapin@gmail.com
Barbara Furlotti, Antiquities in Motion :

From Excavation Sites to Renaissance

Collections, Los Angeles, Getty Research

Institute, 20I9, 292 p. /

ISBN 978ı606o659i4, \$ 8o.

La constitution des grandes collections à la Renaissance est un phénomène bien connu. Pourtant, Barbara Furlotti propose dès l'introduction une approche plus originale, en comparant les pratiques anciennes avec les pillages actuels des sites archéologiques en Italie ou au MoyenOrient. Elle invite ainsi le lecteur à porter le même regard critique, à décentrer le propos pour ne plus penser les collections d'antiques comme une simple activité des élites confortant leur domination sociale et leur capital culturel à l'époque moderne. En adoptant une bottom-up perspective (p.3), l'auteur souhaite retracer en huit chapitres l'itinéraire des objets de leur découverte jusqu'aux collections. Outre l'attention portée à la culture matérielle et aux aspects pratiques, la complexité du tissu socio-culturel est analysée, en mettant l'accent sur des acteurs souvent délaissés comme les paysans ou les restaurateurs. L'expression Antiquities in motion prend alors tout son sens. L'ouvrage s'intéresse au processus amenant une statue de la zone d'excavation à la collection d'un richissime client, en passant par les espaces de vente, de restauration et le transport. Les mobilités au sein de la hiérarchie sociale sont mises en lumière, notamment avec l'enrichissement des découvreurs et des intermédiaires. Le propos s'appuie sur une grande diversité de sources: artistiques évidemment, mais aussi épistolaires, judiciaires, légales, littéraires, papales et privées.

Le premier chapitre, “Brought to Light: Digging in and around Rome», s'intéresse aux conditions matérielles de découverte des sculptures, souvent de manière fortuite, grâce à des vignerons ou à des cultivateurs. L'essor de ces pratiques entraîna 
certes des abus, mais aussi une volonté de régularisation avec un patto di riserva pour tout acte de construction ou de démolition à Rome. Des lois encadrèrent également les activités des fours à chaux. Ces derniers sont théoriquement exclus de la ville en I557. Néanmoins, la législation papale ne permit pas d'éradiquer les pillages de l'Urbs et de contrôler l'ampleur des destructions. Les œuvres excavées intégraient ensuite le marché de l'art analysé dans le deuxième chapitre («Marketing Antiquities : Marketplaces, Companies and Shops»). Ce trafic a un impact visuel fort dans la cité. Le Campo de' Fiori et la Piazza Montanara furent des plaques tournantes du commerce d'antiquités. De multiples acteurs étaient impliqués, comme l'antiquaire Fulvio Orsini. Au-delà des parcours individuels, Barbara Furlotti insiste sur le système entrepreneurial se mettant en place avec des intermédiaires multiples, comme les partenaires Vincenzo Mantovano et Giuseppe Della Porta dans les années i55o. L'auteur étudie alors tout un écosystème allant du pilleur au grand collectionneur. Puis, l'identité, les réseaux et le rôle ambigu des antiquaires sont mis en lumière («Acquiring Meanings, Gaining Value: Antiquarians and the Market for Antiquities »). Une étude de cas de la famille Stampa est proposée. Leurs compétences devinrent une tradition familiale. Deux générations se sont illustrées : le père tailleur constitua une collection au milieu du $\mathrm{xvI}^{\mathrm{e}}$ siècle et ses deux fils furent des marchands renommés. L'auteur revient régulièrement dans l'ouvrage sur cette famille. La définition d'“ antiquaire» est affinée. Certes, il y a des érudits comme Fulvio Orsini, mais ce terme désigne aussi des connaisseurs aux compétences plus techniques, comme le marchand Vincenzo Stampa. Les conséquences des restaurations sont également appréhendées, tant du point de vue de la réalisation matérielle et de la plus-value escomptée que de la réception, y compris sur le long terme avec des statues de-restored (p. II8) au xx siècle
(“From Restoration to Sale: Antiquities in Sculptors' Workshops»). Le travail des restaurateurs répondait à un impératif esthétique qui influença longtemps les sensibilités.

Les deux chapitres suivants, (“The Price of Collecting: Acquiring Antiquities in Sixteenth-Century Rome» et "The Other Side of the Coin: Collecting meets Illegality"), explorent les facettes de l'exploitation commerciale des antiquités, notamment les stratégies déployées pour susciter l'intérêt d'acheteurs éloignés de Rome ou encore l'art de la négociation. Barbara Furlotti en profite pour souligner le rôle essentiel d'agents comme Scipione Sadilli. La part d'ombre de ce négoce est analysée (vol d'antiquités, fraudes...). Les activités illégales furent encouragées par la négligence des autorités et par un contrôle inefficient des fouilles. Le renforcement de la législation papale en matière d'exportation d'antiquités est abordé dans le septième chapitre, “ Exporting Antiquities : The Rules and the Practice », notamment à travers l'action de Paul IV en i556 (un motu proprio pour la protection des antiquités romaines) ou par le biais des licences d'export. Toujours soucieuse de mettre en évidence des pratiques moins conformistes, l'auteure étudie des pratiques alternatives pour contourner les interdictions d'exportation. Enfin, le dernier chapitre, (“ Moving Antiquities Around »), aborde les aspects logistiques du transport des antiquités et les risques encourus, allant de la casse aux tempêtes en mer, en passant par les pirates.

L'ouvrage est richement illustré (I3I figures en couleurs) et propose un carnet de sources en appendice (p. 246-270). À travers l'étude d'exemples précis, Barbara Furlotti n'offre pas seulement un énième ouvrage sur la découverte et l'engouement des grandes figures de l'époque moderne pour les antiquités. Elle met en valeur la culture matérielle et toute la complexité du tissu socio-culturel impliqué, du simple paysan 
trouvant une statue dans son champ aux grands collectionneurs.

Cyrielle Landrea

Université Bretagne Sud cyrielle.landrea@univ-ubs.fr

Silvia Giorcelli Bersani et Filippo CarLàUhink (éds.), Monsieur le Professeur... Correspondances italiennes I853-I888.

Theodor Mommsen, Carlo, Domenico, Vincenzo Promis, Paris, Académie des Inscriptions et Belles-Lettres, 2018, 332 p. / ISBN $9782877543712,35 €$.

Ce volume propose l'édition commentée et admirablement contextualisée d'un dossier épistolaire impliquant Theodor Mommsen et une famille d'érudits turinois de son temps. Il s'agit de la triade glorieuse des Promis, d'où la “Sainte Trinité » dans une lettre de i87o de Mommsen: l'ingénieur, archéologue et épigraphiste Carlo Promis (I8o8-1873), nommé inspecteur des Monuments d'Antiquité, archéologue royal et professeur d'architecture; son frère aîné, Domenico Casimiro Promis (I804-I874), directeur du Médailler et de la Bibliothèque royale; le fils de ce dernier, Vincenzo Promis (I839-I889), qui reprend les responsabilités de son père.

La préface de Marco Buonocore souligne l'intérêt de ce genre de correspondance savante pour la reconstitution de la société dans les régions piémontaise et valdôtaine, lors de la lente définition culturelle del'Italie d'avant et d'après l'unification. Sur le plan intellectuel, on entrevoit la personnalité complexe de l'érudit, de l'homme public et de l'homme privé. Dans le cas particulier de Mommsen, ces échanges épistolaires sont une source pour comprendre sa manière de raisonner et d'opérer dans le domaine de la recherche ecdotique.

L'introduction de Gian Franco Gianotti reconstitue le contexte historico-culturel, avec les amitiés et les inimitiés savantes. Les inscriptions de la Gaule cisalpine constituent la toile de fond de l'histoire d'une collaboration internationale entre la famille Promis et Mommsen, occasionnant un double transfert : d'un côté, la remontée d'informations piémontaises vers Berlin; de l'autre, de nouvelles perspectives d'édition critique, par la transplantation réussie des méthodes philologiques et ecdotiques germaniques appliquées aux textes littéraires et à l'épigraphie du monde romain.

Les rapports de Mommsen avec les savants piémontais et l'Académie des Sciences de Turin, exemple privilégié parmi tant d'autres correspondants italiens et partie d'un projet en cours «L'Edizione nazionale delle lettere di Theodor Mommsen agli Italiani » (cf. M. Buonocore, Lettere di Mommsen agli italiani, I-II, Vatican, 20I7 [Studi e Testi 5r9-520], avec 833 lettres), sont subsumés à l'entreprise monumentale du Corpus Inscriptionum Latinarum, plus précisément à la partie piémontaise du tome $\mathrm{V}$ du $C I L$, dont le second fascicule est édité en i877. Selon les mots de Mommsen, “Turin n'a pas cessé d'être la capitale de l'Italie pour les études sérieuses » (I869), alors qu'il qualifie Carlo Promis de maestro dell'epigrafia Piemontese (I872). Ce livre apparaît en effet après un premier recueil de S. Giorcelli Bersani, "Torino la capitale d'Italie pour les études sérieuses". Corrispondenza Theodor Mommsen-Carlo Promis ", RSI, I24, 2014, 96o-99o, suivi de sa monographie Torino, capitale degli studi seri. Theodor Mommsen e Carlo Promis, Turin, 2014, données qui ont occasionné l'exposition "Carlo Promis e Theodor Mommsen. Cacciatori di pierre fra Torino e Berlino » (20I5), dans le contexte du bicentenaire de la naissance de Mommsen (20I7).

Les trois dossiers épistolaires sont précédés d'un chapitre substantiel sur les rapports de Mommsen avec Turin, dont ses visites ici et en Piémont et l'entrée 
de Carlo Promis à l'Académie de Berlin : les trois Promis furent pour Mommsen des amis et des interlocuteurs savants, des organisateurs de la vie culturelle, des chercheurs insérés dans des réseaux denses, mais aussi des intellectuels engagés pour l'organisation du consensus dynastique. C'est par ailleurs l'époque de l'essor remarquable des recherches antiquisantes, quand se développe sous un jour nouveau le collectionnisme, quand on assiste à l'institutionnalisation de la recherche (associations savantes de storia patria, Académies, musées, universités) et que s'affirme le positivisme, sans oublier les rapports politiques entre Turin et Berlin, dans une Europe en reconfiguration et qui traverse des crises répétées. On dispose ainsi d'un échantillon représentatif de la culture et de la société dans les États de la Maison de Savoie au xIx ${ }^{\mathrm{e}}$ siècle. La question épineuse des tituli falsi vel alieni, cruciale dans le Piémont, avait exigé une prudence particulière: Mommsen déplore une "galerie des faussaires ", ce qui provoque les protestations de Carlo Promis, et la reformulation du premier: "On peut parler plutôt d'une galerie formidable de falsifications que de faussaires en Piemont». L'atmosphère politique conditionne les collaborations savantes, comme il ressort d'une observation à la fois lucide et amère de Mommsen, dans une lettre du 8 septembre i87o : « Mais comment ferons-nous pour renouer les relations avec nos amis en France? après cette guerre horrible et surtout horriblement barbare je ne comprends pas comment on pourra poursuivre les études en commun » (voir en général E. Gran-Aymerich, “Theodor Mommsen (I8I7-Igo3) et ses correspondants français : la "fabrique" internationale de la science », $J S$, 2008, I77-229).

L'ouvrage, édité avec l'aide du département des Études historiques de l'Université de Turin et de l'Institut d'Histoire de l'Université de Potsdam, reproduit des lettres conservées à la Bibliothèque royale de Turin et dans le Nachlass Mommsen de la Bibliothèque nationale de Berlin. Les trois lots de lettres échangées et conservées entre Mommsen et les historiens turinois, au cœur des antiquités régionales, sont très inégaux : 65 lettres avec Carlo Promis (I863-I872), en français; 87 lettres avec Vincenzo Promis (I869-I888), en français et en italien; enfin, seulement 6 lettres avec Domenico Promis (I853-ı869), en français. La correspondance comporte, comme souvent, des lacunes, avec des lots perdus, mais illustre une collaboration prolifique, qui intéressera en particulier les épigraphiques, grâce aux transcriptions d'inscriptions, aux dessins et aux détails généreusement fournis par les trois Promis.

Le recueil, illustré en outre par I9 planches (des photos, des dessins, des reproductions de manuscrits et de lettres), est complété par trois annexes. L'Annexe I, sur Mommsen et la Vallée d'Aoste, présente la naissance des études épigraphiques dans cette région, en grande partie due à Carlo Promis (Antichità di Aosta, 1862) et au savant allemand. L'Annexe II s'intéresse à la “République des lettres» ample et variée, composée d'universitaires, d'élites intellectuelles, d'hommes politiques, de diplomates, d'avocats et de juges, de bibliothécaires et d'archivistes, mais aussi d'éditeurs et de libraires, lors de la création d'une communauté scientifique internationale. Instructif est dans ce sens le rôle du libraire et éditeur Hermann Loescher dans l'internationalisation de la culture turinoise. Les lettres remplissent également d'autres fonctions: parfois, elles sont un moyen de publication scientifique dans les bulletins et les revues; d'autres fois, des lettres d'autres personnes sont transcrites en partie, voire jointes. Une autre vie des lettres apparaît sous nos yeux: “une véritable vie: elles continuaient de se mouvoir, de se faire porteuses d'informations scientifiques, de nouer des rapports sociaux»(p.3II). Enfin, l'Annexe III donne un aperçu 
chronologique du Royaume de Sardaigne et d'Italie, en rapport avec la vie des quatre correspondants. Un index des personnes et un index épigraphique closent le livre.

Cette collaboration autour des "études communes» reste toutefois inégale, en termes d'autorité et de services, d'où le titre du recueil, copiant l'adresse habituelle, très respectueuse, de “Charles » Promis au savant allemand. Des notes explicatives nourries accompagnent l'édition soignée des lettres, mettant à profit une riche bibliographie sur les correspondances scientifiques, les recherches épigraphiques et d'histoire locale. Ce volume paru dans la prestigieuse collection de l'AIBL, où ont été publiés les échanges entre Mikhaïl Rostovtzeff et Franz Cumont (2007), ainsi qu'entre Franz Cumont et Alfred Loisy (20I9), constitue un nouvel exemple du grand potentiel offert par l'exploitation des archives et de la correspondance savante.

Dan Dana

CNRS - HisoMA dan.dana@mom.fr

Christian JАсов, Faut-il prendre les

Deipnosophistes au sérieux?, Paris, Les Belles Lettres, 2020, 3o4 p. / ISBN 978225I45I206, 2 I є.

En ces temps confinés, Christian Jacob nous invite à la table des Deipnosophistes, symposium roboratif pour bibliophiles, philologues, archéologues et historiens. Si l'on dispose d'une abondante bibliographie pour les quinze livres d'Athénée de Naucratis, reflet de l'incroyable richesse documentaire de l'œuvre, l'historien propose une enquête originale, "quasi ethnographique ", construite à partir de l'hypothèse que le cercle d'érudits, mis en scène par l'auteur autour du chevalier romain Larensis, fait référence à un cercle réel. Il présente aussi ce double intérêt de donner des clés pour naviguer dans cet hypertexte et d'apporter un éclairage sur la culture, les rôles des bibliothèques, compilations et citations dans la construction et la transmission des savoirs livresques à l'époque impériale.

L'enquête s'ouvre sur la mise en exergue de ce qui fait la spécificité générique des Deipnosophistes et leur étrangeté absolue pour un lecteur d'aujourd'hui : la logique taxinomique de la compilation ancrée dans la forme discursive du dialogue qui permet de déployer un réseau complexe de propos et de citations se prolongeant en différentes ramifications thématiques. Il est donc nécessaire de comprendre le projet général d'Athénée pour pleinement saisir la composition de cette œuvre, « bibliothèque mise en livre » (p. 33). Par conséquent, après avoir rappelé la structure des livres, dont le découpage suit le rythme des conversations entre les convives du festin, Christian Jacob les replace dans la tradition de la littérature symposiaque pour en souligner la dimension réflexive, l'œuvre reconstituant la pratique sociale du banquet tout en offrant une réflexion polysémique sur les savoirs et sources sur le banquet, comprenant pas moins de 800 auteurs et 2500 œuvres. Si nous ignorons quasiment tout d'Athénée et que les incertitudes persistent sur le cercle d'intellectuels évoluant autour de Larensis, se dessine néanmoins un espace culturel triangulaire, reliant Athènes, Alexandrie et Rome par l'onomastique, l'origine et le milieu de l'auteur. De Platon à Plutarque, les récits rapportés par Athénée à Timocrate s'inscrivent dans cette tradition du banquet comme rite de sociabilité et espace de convivialité : comme le vin et les plats, la culture et les savoirs circulent et se partagent à ces tables de fiction.

Ces prémices posées, l'historien s'attache à analyser le cercle d'érudits ainsi rassemblé. Larensis est l'hôte raffiné d'un “ cénacle cosmopolite» (p. 47), reflet des élites évoluant dans la Rome impériale du $\mathrm{II}^{\mathrm{e}}$ siècle et foyer intellectuel dans la tradition 
alexandrine. Parmi eux, se trouvent d'ailleurs Philadelphe de Ptolémaïs, Plutarque d'Alexandrie, Ulpien de Tyr et l'illustre Galien. L'historien souligne le problème de l'identification des convives, d'où deux hypothèses: celle d'un cercle réel sous Septime Sévère ou celle d'un cercle fictif associant des figures passées et présentes, sans pouvoir définitivement trancher. Les noms des vingt-deux convives mis en scène permettent toutefois de condenser un microcosme représentatif de la paideia, de la culture et des savoirs contemporains, tel un nouveau Museion. La structure de l'œuvre est ensuite précisée : le banquet chez Larensis est raconté par Athénée à Timocrate qui n'a pu y assister, procédé narratif réactualisant le festin et faisant de l'interlocuteur, et donc du lecteur, un deipnosophiste. L'auteur en analyse les principes ordonnateurs: le découpage en quinze livres et en dialogues, un séquençage suivant les tempi du festin, une organisation thématique, autant d'éléments construisant une architecture complexe au-delà du flot des propos. Le système énonciatif et sa mise en scène théâtrale permettent de faire dialoguer convives et livres, suivant un “processus de réactivation de la mémoire culturelle» (p. 7i). Un autre intérêt de l'essai est alors de replacer la bibliothèque de Larensis dans le contexte des grandes collections de livres antiques. Ce cercle de bibliophiles témoigne de leur quête incessante de livres, de leur connaissance fine des sources citées, de la précision de leurs références bibliographiques, d'un état des savoirs et de la transmission des textes à l'époque impériale. Ce savoir bibliographique dessine une carte de l'érudition lettrée héritée de la tradition hellénistique: les convives philologues et lexicographes déploient un ensemble de techniques permettant d'y circuler, telles que la lecture experte par la prise de notes, la mémorisation de citations impliquant des jeux mnémotechniques et le système agonistique de questionnement entre les interlocuteurs.

Un dernier temps de l'ouvrage apporte un éclairage décisif sur le projet auctorial, la forme et les significations de l'œuvre. Au-delà de la compilation, il s'agit bien de proposer une structure thématique régulée par l'orientation donnée aux échanges par les convives, ce qui permet à l'auteur de donner une cohérence à l'ensemble des informations que recèle le texte et à son destinataire de construire un parcours de lecture et de naviguer dans l'œuvre. C'est un lectorat restreint auquel s'adresse Athénée en un "texte-miroir " (p. I76) à la bibliothèque de Larensis. Les Deipnosophistes se font, sur le mode ludique des interactions entre les convives, un hypertexte permettant de naviguer dans un réseau d'hypotextes suivant un fil conducteur qui peut être thématique, lexical ou narratif. Christian Jacob en souligne la double logique, centripète et centrifuge, transformant le texte en épitomé qui condense un monde de livres, dans lequel le lecteur est invité à circuler en une véritable périégèse des savoirs. Cela conduit l'historien à matérialiser le projet par la comparaison avec l'architecture du Gugenheim Museum de F. Lloyd Wright, ce qui lui permet de distinguer quatre niveaux de lecture en un dispositif hélicoïdal : la bibliothèque comme espace de navigation, la structuration bibliographique, la métalittérature des périodes hellénistique et impériale, un niveau à l'échelle de la culture et des savoirs circulant à Rome à la fin $d u{ }{ }^{\mathrm{e}}$ siècle et un ultime comme anamnèse culturelle, faisant de l'œuvre d'Athénée le miroir des préoccupations d'une civilisation s'interrogeant sur son identité et ses valeurs. Ces niveaux de lecture conduisent in fine à réinterroger le statut de l'auteur et du lecteur. Athénée, en compilateur, bibliophile érudit et "père du livre ", est à la fois narrateur interne et lecteur mettant en abyme la poikilia des textes, qu'il a lus et cite, et en 
scène la bibliothèque qu'il côtoie, la figure auctoriale se faisant dès lors l'ordonnateur des références citées et glosées. Lecteur du texte, interlocuteur d'Athénée et auditeurs $\mathrm{du}$ banquet constituent trois figures de lecteur d'un texte qui compile et organise des notes et citations et sollicite leur remémoration et leur oralisation à la façon d'une recitatio, et suivant un mode réflexif puisqu'il prend pour objet le banquet luimême. Cette mise en abyme est renforcée par la polysémie de termes comme pinakes, plateaux pour le banquet et tables lexicales, ou paratithesthai signifiant servir et citer, si bien que « les extraits du texte participent du même geste d'ostentation, et peut-être du même processus de préparation et de présentation, entre l'art et la technique, par découpage, conditionnement, assemblage et assaisonnement de matières premières, naturelles ou linguistiques »(p. 267). Ces considérations amènent l'historien à conclure sur la séduisante hypothèse que les Deipnosophistes constitueraient le récit d'un jeu de société reposant sur un parcours transversal dans la bibliothèque, à travers un échange de questions et de citations entre les participants, jeu auquel nous fait indirectement participer Christian Jacob au terme de cette passionnante exploration de la carte et du territoire de l'œuvre d'Athénée.

Clarisse Evrard

Université de Lille clarisse.evrard@univ-lille.fr

\begin{abstract}
Annelies Lannoy, Alfred Loisy and the Making of History of Religions. A Study of the Development of Comparative Religion in the Early 2oth century, Berlin et Boston, De Gruyter, 2020, 366 p. / ISBN 9783іго583779, 86,6оє.
\end{abstract}

Comme nous le rappelle l'exergue de ce beau livre, Alfred Loisy (I857-I940) se prenait pour «l'Einstein de l'histoire des religions », " pour avoir dit que la vérité des Écritures était relative ». On se prend à sourire. Pour s'en tenir aux chercheurs catholiques l'ayant précédé, nommons au moins l'oratorien Richard Simon (I638-I7I2), ou même son maître Ernest Renan, mort quand Loisy avait trente-cinq ans - sans compter que l'étude critique des textes bibliques ne représente pas vraiment toute l'histoire des religions. Prêtre excommunié en I9o8, et élu à la chaire d'histoire des religions du Collège de France l'année suivante, il y enseignera pendant de longues années. C'est cependant plus pour son rôle décisif dans la formation du mouvement moderniste dans le catholicisme français que pour son approche des phénomènes religieux que Loisy est passé à la postérité. On peut citer à ce sujet sa phrase célèbre : “Jésus annonçait le Royaume, et c'est l'Église qui est venue. »

Dans un ouvrage novateur, qui fait le point sur le personnage scientifique de Loisy, Annelies Lannoy se propose de cerner de façon précise le personnage scientifique de Loisy, pour comprendre, en suivant les péripéties majeures de sa carrière, à travers son évolution intellectuelle, sa contribution à la discipline de l'étude historique des religions. En cinq riches chapitres, Lannoy fournit des analyses méticuleuses, en s'appuyant non seulement sur les écrits publiés de Loisy et de ses contemporains, mais aussi sur sa correspondance (par exemple avec des savants tels que Franz Cumont et Salomon Reinach).

L'auteure part de L'Évangile et l'Église (I902), qui, annonçant la crise moderniste, représente la réponse de Loisy aux vues de Harnack sur la spécificité incomparable du christianisme (Das Wesen des Christentums [I9or]), montrant que, déjà, Loisy insiste pour comprendre la naissance du christianisme dans le cadre des religions du monde méditerranéen et proche-oriental : judaïsme et hellénisme avant tout. L'élection de Loisy au Collège de France, objet du 
second chapitre, peint de couleurs vives le délicat ballet, ou, si l'on préfère, l'imbroglio, entre projets scientifiques, attitudes religieuses, préférences politiques, préjugés et antipathies personnelles qui semble être la règle (en tous cas à l'époque) dans cette auguste institution. Le chapitre trois insère Loisy dans le contexte des relations entre magie, science et religion dans la France du début du $\mathrm{xx}^{\mathrm{e}}$ siècle. Reinach, porte-parole de l'anthropologie britannique, et Mauss (qui perdit face à Loisy l'élection au Collège en 1909) sont ici les interlocuteurs privilégiés. Le rejet $\mathrm{du}$ "mythe du Christ», et Les mystères païens et le mystère chrétien (I9I9) font l'objet du chapitre quatre. Lannoy montre comment c'est grâce à la théorie (venue d'Angleterre) du ritualisme que Loisy réfute l'idée (saugrenue mais largement diffusée à l'époque) que la personne de Jésus n'avait rien d'historique. Dans un dernier chapitre, ce sont les recherches comparatives (menées pendant plusieurs années dans ses cours au Collège de France) sur l'histoire et l'idée du sacrifice qui sont étudiées. Lannoy montre bien comment l'approche de Loisy sur le sacrifice s'oppose aux conceptions d'Hubert et de Mauss.

S'il fallait définir le genre dans lequel s'inscrit l'ouvrage, on pourrait parler de micro-histoire intellectuelle et institutionnelle, en faisant allusion à la microstoria pratiquée avec verve, dans l'Italie des années i97o, par Giovanni Levi, Carlo Ginzburg et Simona Cerutti. On apprend vraiment beaucoup sur Loisy et sur son œuvre, tous deux réintégrés dans leurs milieux intellectuel, religieux, politique et institutionnel. Ce qui frappe avant tout, c'est la complexité de l'agencement de ces milieux. Loisy évolue entre les chercheurs catholiques, protestants, juifs, catholiques hérétiques, comme lui, libre penseurs, laïcs, agnostiques et athées. Les dimensions européennes de la recherche en histoire des religions vers la fin du $\mathrm{xIx}^{\mathrm{e}}$ siècle et dans les premières décennies $\mathrm{du} \mathrm{xx}^{\mathrm{e}}$ siècle, abordées par le biais du héros dans ce livre, montrent à quel point la France (ou plutôt Paris) fait exception. L'histoire des religions prend son essor institutionnel, en France, dans le troisième tiers du xIx ${ }^{\mathrm{e}}$ siècle, avec la création d'une chaire d'histoire des religions au Collège de France et de la Section des Sciences Religieuses à l'École Pratique des Hautes Études. Le rôle des chercheurs protestants, et aussi des chercheurs juifs, est ici fondamental : nulle part ailleurs, de l'autre côté du Rhin ou de la Manche, on ne trouve des savants de différentes identités religieuses (ou des libres penseurs) travaillant ensemble, avec les mêmes méthodes, en histoire comparée des religions. Une telle situation exceptionnelle de la France devait permettre le développement d'approches nouvelles. Par exemple, à la fois catholiques et juifs insistent sur le rôle central du rite, plutôt que, comme les protestants, sur la foi, pour définir l'essence de la religion. Pourtant, ce statut et ce rôle particuliers de la France dans la discipline sont trop souvent passés sous silence, ou restent mal compris.

On est en droit de se demander pourquoi l'impact de Loisy, en France tout autant qu'à l'étranger, restera somme toute mineur. La réponse à cette question, seulement esquissée par Lannoy, me semble être liée, au moins en partie, au fait que c'est la naissance du christianisme qui reste le cœur de l'intérêt de Loisy. Or les études néotestamentaires, développées surtout en milieu protestant, ne jouent nulle part un rôle important dans la discipline de l'histoire comparée des religions. La figure intellectuelle de Loisy, qui maintenant, grâce à Lannoy, se profile clairement, est incontournable pour qui veut mieux comprendre l'histoire de la discipline.

Guy G. Stroumsa Université d'Oxford, Université Hébraïque de Jérusalem guy.stroumsa@mail.huji.ac.il 
Annelies Lannoy, Corinne Bonnet et Danny Praet (éds.), “Mon cher Mithra ». La correspondance entre Franz Cumont et Alfred Loisy, 2 vols., Paris, Académie des Inscriptions et Belles-Lettres, 2019, 448 p. et 646 p. / ISBN 9782877543743 , 120 €.

Franz Cumont (I868-I947) et Alfred Loisy (I857-I940) comptent parmi les grands spécialistes d'histoire religieuse $\mathrm{du} \mathrm{xx}^{\mathrm{e}}$ siècle, le premier dans le domaine des religions orientales dans le monde romain, le second au sujet des origines du christianisme. Leur parcours académique n'est pas comparable. Cumont est un enseignant de l'université de Gand. Loisy est ordonné prêtre en I879. Exégète, il est au cœur de la querelle moderniste qui lui vaut l'excommunication en rgo8 et l'admission au Collège de France l'année suivante. Mais les deux hommes vont entretenir une importante correspondance qu'une publication de l'Académie des Inscriptions et Belles-Lettres, sous la responsabilité de Annelies Lannoy, Corinne Bonnet et Danny Praet, offre au public en deux volumes. La totalité de cette correspondance représente 409 lettres allant du 8 mai igo8 au io mai I940. Le premier volume, agrémenté d'une belle introduction présentant les biographies des deux intellectuels, le contexte, les enjeux intellectuels, politiques, idéologiques liés à la correspondance, regroupe l'ensemble des missives. Le second propose aux lecteurs une étude critique érudite des lettres. L'ensemble met à la disposition du public une documentation passionnante sur plusieurs plans. Tout d'abord, les échanges intellectuels entre deux savants. Par exemple, le 24 août I9I9, Loisy écrit à Cumont au sujet de saint Ambroise : ‘ J'avais bien vu que votre texte de saint Ambroise ne se rapportait pas à une ascension réelle. Par rapport à celle-ci l'ascension morale est une façon de transposition. Si Ambroise ne parle que de la dernière, c'est qu'il n'a pas trouvé autre chose dans ses sources. Comme il pille volontiers volontiers (sic)
Origène, c'est peut-être là qu'il aura pris son idée de l'ascension morale contrariée par les puissances de l'air, Origène n'ayant pas voulu admettre autre chose. Mais je ne suis pas en état de vérifier cette conjecture ».

Ensuite, sur les effets de la crise moderniste qui traverse le monde catholique au début du $\mathrm{xx}^{\mathrm{e}}$ siècle mais dont les effets ont duré jusqu'au pontificat de Jean XXIII. Les travaux d'Emile Poulat jusqu'à ceux de Maurilio Guasco et Pierre Colin nous permettent de mieux comprendre cette crise majeure de la pensée catholique. Le 2 avril I9ı3, Cumont en présente les enjeux : “ Vous ne serez compris ni de ceux qui acceptant aveuglément une théologie traditionnelle, ni de ceux qui prétendent à l'aide de notre pauvre science résoudre toutes les énigmes de l'univers. On vous lapidera de droite et de gauche, on vous accusera à la fois de blasphème et de cléricalisme, mais vous possédez mieux que vos adversaires l'intelligence de ce qu'est la religion et de ce qu'elle devrait être, vous avez acquis en mangeant du fruit défendu la connaissance du bien et du mal, et tous ceux que n'aveuglent pas de préjugés héréditaires ou contemporains, vous suivront avec sympathie, et s'instruiront en vous lisant ».

Puis, un éclairage sur l'actualité de l'époque de la correspondance. Ainsi, l'impact de la Première Guerre mondiale qui fracture la communauté scientifique et sur laquelle les jugements portés ne manquent pas de pertinence par moments. Le 24 septembre I9ı5, Cumont écrit à Loisy que “les Bougres vont probablement allumer un nouvel incendie dans les Balkans. On aurait pu l'empêcher de s'allumer, il sera difficile de l'éteindre. La guerre se propage au lieu de se circonscrire et les dévastations se font plus atroces. C'est évidemment un remède radical à la surpopulation, mais c'est aussi le suicide de l'Europe. L'Amérique sera le tertius gaudens, appelé à recueillir les fruits de notre labeur passé ». De même, la condamnation de l'Action 
française par le pape Pie XI en I926. Le 25 novembre I927, Loisy écrit à Cumont: “ Pie XI est à sa manière un gardien aussi rigide de l'orthodoxie que l'était feu Pie X. L'Action française s'en est encore aperçue ces jours-ci. Je ne sais si vous avez lu les instructions données par la Pénitencerie aux évêques français sur ordre du pape. On veut entièrement désaffecter la chapelle de Maurras. Il faudra choisir entre elle et l'Église ».

La présente publication est donc un outil passionnant mis à la disposition de ceux qui travaillent sur l'historiographie de l'histoire ancienne et sur l'histoire culturelle de la période contemporaine.

Philippe Foro

Université Toulouse - Jean Jaurès philippe.foro@wanadoo.fr

\section{Jonatan Pérez Mostazo, Lustrando las raíces. Antigüedad vasca, política e identidades en el siglo xIX, Pamplona, Urgoiti Editores, 2019, 496 p. / ISBN 97884121036oI, $20 €$.}

Le livre de Jonatan Pérez Mostazo, publié par la maison d'édition Urgoiti, bien connue de tous ceux qui s'intéressent à l'histoire de l'historiographie espagnole, est issu d'une thèse de doctorat préparée sous la direction d'Antonio Duplá Ansuátegui et soutenue en 2018 à l'Universidad del País Vasco (Vitoria-Gasteiz). Il prend pour objet d'étude l'Antiquité basque (le territoire envisagé est défini en introduction, p. I5-i6) et l'ensemble des représentations auxquelles elle a donné lieu à l'époque contemporaine en privilégiant les sources du $\mathrm{XIX}^{\mathrm{e}}$ siècle. Lustrando las raíces est donc un livre sur la réception de l'Antiquité dans la culture historique des provinces basques espagnoles historiques (la Biscaye, l'Álava, le Guipúzcoa, la Navarre ; le cas des territoires français du Labourd, de la Basse-
Navarre et du Soule ne sont pas traités). Il permet de répondre à deux questions principales : quelle place occupe l'Antiquité dans les projets politiques et dans les discours identitaires élaborés tout au long du XIX ${ }^{\mathrm{e}}$ siècle? Comment s'adaptent-ils à des contextes politiques et historiographiques mouvants qui, à chaque nouvelle étape, donnent lieu à une actualisation du récit afin que ce dernier réponde aux préoccupations d'une élite déterminée dans un contexte précis?

L'auteur l'affirme avec force dès l'introduction: "L'utilisation de l'expression [Antiquité "basque"] ne répond pas à une conception essentialiste de l'histoire; on refuse catégoriquement l'existence d'une réalité territoriale ou humaine qui, à l'époque ancienne, pourrait être désignée comme basque » (p. I4). Le tableau que nous offre Jonatan Pérez Mostazo est à la fois complexe et nuancé. Les discours produits sont pluriels, rarement consensuels, souvent contradictoires et se répondent les uns les autres. D'une façon ou d'une autre, tous sont producteurs de mythe, même les - rares - textes qui tentent d'assimiler les acquis d'une science historique méthodique dans les dernières décennies du siècle. Sans jamais basculer dans le jugement de valeur et en s'efforçant toujours - avec succès - de maintenir à distance son objet d'étude, l'auteur mène une entreprise de déconstruction : il s'agit de comprendre les multiples avatars du mythe selon lequel les Basques, en n'ayant jamais été complètement romanisés, seraient restés à travers les siècles un peuple au particularisme affirmé, préservé des influences extérieures et radicalement différent du reste des Espagnols.

L'approche s'appuie sur un jeu d'échelles. L'auteur analyse les discours produits par une élite intellectuelle et politique basque qui puise dans l'Antiquité des arguments visant à justifier son discours identitaire national. S'il laisse de côté les travaux des voyageurs et des érudits étrangers, le 
livre fait dialoguer en permanence cette production historiographique avec la vision qui s'élabore depuis la capitale espagnole. Entre le régional et le national, les liens établis sont complexes. Les réponses que les savants madrilènes (en particuliers les membres de la Real Academia de la Historia) adressent aux lettrés des provinces basques ne se réduisent pas à un rejet de leur discours historique. La vision de l'Antiquité promue par l'historiographie basque est tantôt combattue, tantôt soutenue, tantôt ignorée par les membres des principales institutions académiques madrilènes.

Les œuvres étudiées et les polémiques qui en découlent montrent l'extrême malléabilité des sources, qu'il s'agisse des textes transmis par les Anciens ou des restes matériels du passé. Le registre archéologique prend une importance particulière à partir des années I870-I880 grâce à l'action des Comisiones Provinciales de Monumentos. En identifiant et en permettant de mieux connaître les vestiges de l'époque antique, les travaux de ces institutions fragilisent, sans parvenir à les détruire, les théories selon lesquelles les provinces basques n'auraient connu qu'une faible romanisation.

D'un auteur et d'une période à l'autre, le débat s'organise toujours autour des mêmes enjeux. Il s'agit de puiser dans le passé des arguments destinés à défendre ou à condamner l'existence des privilèges administratifs et juridiques dont bénéficient les provinces basques au sein de l'État espagnol (fuerismo). Pour les partisans de ce particularisme, l'idée selon laquelle les Basques de l'Antiquité auraient maintenu leur indépendance face à Rome et auraient négocié volontairement leur intégration à l'empire romain puis à la couronne de Castille suffit à défendre les fueros. Dès lors, les questions auxquelles les érudits tentent de répondre sont toujours les mêmes: à quelle ethnie mentionnée par les auteurs de l'Antiquité fallait-il assimiler les Basques contemporains? Aux
Cantabres qui avaient farouchement résisté à Rome (vascocantabrismo)? Aux Vascons? Ces populations furent-elles soumises à Rome? À quel moment de leur histoire? La langue basque, l'euskara, existait-elle dans l'Antiquité ? Était-elle l'héritière directe de la langue des Ibères (vascoiberismo)?

La première partie du livre, composée de trois chapitres (le sommaire est téléchargeable sur le site de l'éditeur: http://www.urgoitieditores.com/libros/ monografias/lustrando-las-raicesantiguedad-vasca-politica-e-identidadesen-el-siglo-xix/), étudie la place de l'Antiquité dans les discours politique et identitaire qui se développent entre la fin du $\mathrm{xVIII}^{\mathrm{e}}$ et le début du $\mathrm{xx}^{\mathrm{e}}$ siècle. L'étude prend comme point de départ la tradition historiographique élaborée à la fin de l'époque des Lumières par Enrique Flórez et Manuel Risco. À partir de là, les différents récits élaborés sont rigoureusement situés dans leur contexte politique, social, culturel et intellectuel. L'analyse est chronologique et progresse au rythme des grandes coupures de la vie politique espagnole. Les Lumières et la rupture née de l'invasion napoléonienne et de la guerre d'indépendance, alors que se développe le premier projet politique libéral et sa réponse contre-révolutionnaire, germe du futur mouvement carliste, sont étudiées dans le chapitre I.

L'historiographie romantique de l'époque isabelline est abordée dans le chapitre 2. Marquée à ses débuts par la première guerre carliste, elle voit ensuite prendre forme un projet politique de défense du particularisme local compatible avec le nouvel État libéral - dominé par un libéralisme modéré soucieux de faire la synthèse entre les principaux acquis révolutionnaires d'un côté et, de l'autre, l'ordre et la tradition - (défense des fueros, de la religion catholique, de l'euskara et d'un double patriotisme, basque et espagnol). 
À l'époque de la Restauration (chap. 3), les traditions antérieures, encore dominées par l'antiquarisme, restent vivaces mais le panorama historiographique devient plus complexe. À la fin du siècle se développe une nouvelle approche du passé régional influencée par une méthode positiviste qui tente de déconstruire les mythes solidement ancrés dans de larges secteurs de la population, sans parvenir à y renoncer complètement. Ces avancées sont timides comme le montre la citation que l'auteur donne de l'appel lancé par Fidel Fita dans le Boletín de la Real Academia de la Historia, en I899, pour " détruire les fausses idoles suscitées par l'ignorance (innocente il y a deux siècles, coupable aujourd'hui)» et pour élaborer un récit fondé sur " des documents et des monuments positifs, et non sur des châteaux de cartes ou des analogies chimériques »(p. 236). D’autre part, les années I8go voient surgir le discours identitaire nationaliste élaboré par Sabino Arana. Encore faiblement implanté parmi la population basque, il est bien plus excluant, marqué par le désir d'accentuer le particularisme des provinces basques en rejetant tout lien historique avec le reste de l'Espagne, au point de les présenter “ comme une île dans le devenir historique péninsulaire et européen » (p. 2I7). Les théories du fondateur $\mathrm{du}$ nationalisme basque recueillent l'héritage de l'historiographie de l'époque romantique et les acquis récents de l'anthropologie physique telle qu'elle se développe dans la deuxième moitié du xix ${ }^{\mathrm{e}}$ siècle. Son discours identitaire repose sur quatre piliers que sont la race basque (radicalement opposée aux maketos, les non-Basques), la langue (symbole de l'originalité, de la pureté de la race et instrument privilégié de son isolement face aux influences nocives venues de l'extérieur, synonymes de dégénération), la tradition juridique forale et l'intégrisme religieux. Le projet de Sabino Arana vise à récupérer une indépendance perdue en obtenant la création d'une confédération indépendante des États basques. Sans être au centre de son discours, l'Antiquité y trouve sa place: elle est présentée comme un âge d'or synonyme d'indépendance et d'un particularisme marqué par rapport au reste de la péninsule, ce qui aurait permis au peuple basque de préserver la pureté de son sang à la différence du reste des Espagnols précocement influencés par de multiples influences extérieures.

La deuxième partie du livre (trois chapitres) offre un complément indispensable. L'approche y est cette fois thématique, l'auteur revenant sur les principaux sujets qui ont nourri les discours identitaires et politiques élaborés tout au long du $\mathrm{xIx}^{\mathrm{e}}$ siècle. Jonatan Pérez Mostazo montre que les ressorts sur lesquels ils reposent n'ont rien d'original. Ce sont les mêmes que ceux qui alimentent n'importe quel discours identitaire. Le cas basque est ainsi rigoureusement contextualisé, systématiquement comparé au processus de construction des identités nationales que connaissent au même moment l'Espagne, la France, la Grande-Bretagne ou l'espace germanique. Ajoutons que cette partie fait dialoguer en permanence les sources anciennes et les textes contemporains.

Le chapitre 4 aborde la question de la recherche des ancêtres visant à doter les Basques d'une généalogie ancienne et prestigieuse. Elle postule l'existence d'un lien direct entre les populations contemporaines et celles de l'Antiquité. En donnant à voir la grande hétérogénéité ethnique de la péninsule dans l'Antiquité, le manque de cohésion entre ses différents peuples et leur rythme d'intégration différencié au monde romain, les sources anciennes ont facilité le processus d'identification entre une région et un peuple concret (Cantabres et Vascons / Basques). Le vascocantabrismo perdure au moins jusque dans les années i88o. S’il recule alors dans la culture savante sous l'effet d'une historiographie plus critique 
face aux sources, il reste durablement ancré dans la culture populaire et l'enseignement.

L'idée de nation repose par ailleurs sur un passé héroïque, sur des grands hommes et des hauts faits mémorables (chap.5). Concernant l'identité basque, ce récit s'appuie avant tout sur des exempla fournis par les conflits armés qui ponctuent la conquête romaine. La participation des provinces basques aux guerres civiles de l'époque républicaine est peu utilisée au $\mathrm{XIX}^{\mathrm{e}}$ siècle, à l'exception de l'épisode du siège de Calagurris. Deux moments sont privilégiés : la participation des Cantabres et des Vascons à la Deuxième Guerre punique aux côtés des Carthaginois (on puise abondamment dans l'épopée de Silius Italicus) et les Guerres cantabres (Florus, Orose et Dion Cassius). Ces images sont d'une grande plasticité. Parfois destinées à témoigner du patriotisme des Basques qui sont ainsi associés aux hauts faits de la nation espagnole, elles servent aussi le discours de ceux qui cherchent à convertir les Basques en de farouches défenseurs de leur indépendance luttant contre l'envahisseur étranger.

Le dernier chapitre pose la question de l'influence exercée par Rome sur les provinces basques en soulignant le paradoxe des discours auxquels elle a donné lieu : elle est tantôt synonyme d'intégration au monde civilisé, tantôt symbole de la liberté perdue et de la soumission à une puissance étrangère. Cette question va de pair avec la définition d'une essence basque qui aurait été préservée à travers les siècles. Ces traits particuliers sont extraits des sources anciennes qui fournissent des informations ethnographiques sur les peuples de la péninsule dans l'Antiquité. Mais ces témoignages sont rares, brefs et parfois contradictoires, ce qui conduit à une lecture sélective de ces textes en idéalisant leur contenu pour servir les discours identitaires contemporains qui établissent un lien direct entre les usages et les coutumes des Basques et ceux de leurs ancêtres (caractère belliqueux et attachement à leur indépendance, danse, chant, langue, monothéisme primitif annonciateur du christianisme, etc.). L'idée qui domine est celle du rejet de tout apport de Rome à la culture locale qui aurait au contraire été préservée. Toutefois, dans les dernières décennies duxix ${ }^{\mathrm{e}}$ siècle, marquées par une ouverture de l'Espagne sur le reste de l'Europe, on voit se développer une vision plus positive de la romanisation. Les discours sur l'indépendance basque font alors l'objet d'une relecture. Ils deviennent synonymes d'un isolement négatif face aux principaux courants civilisateurs, tandis que les images idéalisées des ancêtres sont rapprochées d'un stade indésirable de barbarie (p. 386).

$$
\begin{array}{r}
\text { Grégory Reimond } \\
\text { Université de Toulouse-Jean Jaurès / } \\
\text { Casa de Velázquez } \\
\text { gregory.reimond@casadevelazquez.org }
\end{array}
$$

Anna Peterson, Laughter on the fringes. The Reception of Old Comedy in the Imperial Greek World, Oxford et New York, Oxford University Press, 20I9, 240 p. / ISBN 9780I90697099, £64.

Dans l'introduction de cet ouvrage consacré, comme l'indique le titre, à la réception de l'Ancienne Comédie dans le monde grec impérial, Anna Peterson annonce que son étude portera sur cinq auteurs (Plutarque, Aelius Aristide, Lucien, Alciphron et Libanios) et n'a pas l'ambition de fournir une étude exhaustive des références à l'Ancienne Comédie durant toute la période envisagée. Un deuxième élément de cette introduction consiste à rappeler que cette réception est tributaire à la fois de la philologie hellénistique et des critiques émises par Platon et Aristote à l'époque classique. 
Chez Plutarque, premier auteur étudié, on peut observer deux stratégies différentes: sa Comparaison d'Aristophane et Ménandre, dans le droit fil de la Poétique d'Aristote, condamne la grossièreté d'Aristophane, au profit de la comédie plus raffinée de Ménandre; en revanche, dans la Vie de Périclès, l'Ancienne Comédie est utilisée comme une source littéraire et historique significative. Elle permet par exemple de rapporter des allusions aux frasques sexuelles dans l'entourage de Périclès et d'Aspasie. Mais l'autrice ne cite pas le cas d'Hermippos, auteur comique qui aurait intenté un procès à Aspasie. Or ce passage, même si l'authenticité historique n'en est pas démontrée, suggère, ce que confirme d'ailleurs l'ensemble de la Vie de Périclès, que Périclès avait plus à craindre des menées de ses adversaires au moyen des tribunaux et de l'assemblée des citoyens que des plaisanteries traditionnelles des comiques dans un cadre de licence festive.

À propos d'Aelius Aristide, le deuxième auteur examiné, l'autrice étudie trois discours dont le plus important, et à mon sens le plus problématique, est le texte intituléPeri tou mê deîn kômôideîn, titre que l'on traduit d'ordinaire Sur l'interdiction de la comédie. Selon Anna Peterson, l'auteur réagirait à des reprises, sur la scène théâtrale de Smyrne, de pièces de l'Ancienne Comédie. Cette interprétation me paraît discutable. Rien dans le texte n'indique que l'auteur vise la comédie en tant que représentation et genre littéraire. Le verbe kômôideîn signifie de façon générale “ railler, moquer » et surtout le terme kômos (§ 4) nous oriente plutôt vers des défilés de fêtards chantant des satires à l'occasion de fêtes telles les Dionysies. En outre, au $\S 27$, la comédie fait partie des “écrits » (syggraphai) laissés par les Athéniens. Dans son édition de 1898 , Bruno Keil songeait à des satires visant personnellement Aelius Aristide, qui se signalait par sa suffisance et sa superstition. Sans aller aussi loin, on pourrait envisager des satires visant des membres de l'élite smyrniote, ce qui suffit à expliquer la mauvaise humeur d'Aelius Aristide. Ce genre de manifestations était bien plus fréquent que d'hypothétiques reprises de vieilles pièces et c'est un phénomène analogue qui, deux siècles plus tard, suscita la colère de l'empereur Julien contre les habitants d'Antioche et l'amena à composer le Misopôgôn.

Le troisième auteur étudié, Lucien, est sans doute celui chez lequel la réception de l'Ancienne Comédie produit les effets les plus intéressants. Dans le Pêcheur (Anabiountes $\hat{e}$ halieus), le cadre narratif s'inspire des Dèmes d'Eupolis, pièce perdue mais dont on connaît l'intrigue. Dans les Dèmes, quelques grandes figures du passé sortaient des enfers pour être confrontées à la décadence de l'Athènes contemporaine d'Eupolis. Dans le Pêcheur, Lucien imagine que les fondateurs des écoles philosophiques, sortis des enfers, veulent se venger de Parrhésiadès-Lucien qui les a moqués dans Philosophes à vendre (Biôn prâsis). De plus, l'imitation des Dèmes d'Eupolis est combinée avec l'imitation, dans les Acharniens d'Aristophane, du combat entre Dikaiopolis et le chœur des charbonniers, transposé dans le Pêcheur en un débat entre Parrhésiadès-Lucien et les fondateurs des écoles philosophiques.

Une deuxième partie de l'étude de Lucien porte sur la Double accusation (Dis katêgoroumenos). Ce titre renvoie au fait qu'à la fin du dialogue la Rhétorique accuse le Syrien (Lucien) de mauvais traitement (kakôsis) pour l'avoir abandonnée au profit du Dialogue (philosophique). À son tour le Dialogue accuse le Syrien d'hybris pour l'avoir mélangé à la satire et au comique. Selon l'autrice, cette situation évoque la Pytinê (Dame-jeanne) de Cratinos, pièce perdue dont on connaît l'intrigue grâce aux scholies des Cavaliers d'Aristophane. Dans cette pièce Aristophane se moquait de la déchéance de son rival, provoquée par son penchant pour la dame-jeanne. Mais Cratinos produisit alors une pièce brillante, 
où il imaginait que sa femme, la Comédie, lui reprochait de l'avoir délaissée pour la dame-jeanne, et qui gagna le premier prix, surclassant les Nuées d'Aristophane. Mais il faut aussi rappeler que la double accusation finale est longuement précédée de cinq procès qui impliquent une tradition biographique relative aux philosophes du passé et dont on trouve un parallèle chez Diogène Laerce. De ce fait, le souvenir de la Dame-jeanne de Cratinos, tout en étant possible, reste moins nécessaire que la réminiscence des Dèmes d'Eupolis dans le Pêcheur.

Dans une troisième partie sont examinées quelques prolaliai de Lucien, brefs discours introduisant des discours plus développés. La prolalia la plus intéressante est le texte intitulé Tu es un Prométhée en discours (Promêtheus ề en logois). Réagissant à des commentaires sur la nouveauté de ses œuvres, Lucien fait valoir que, plus que la nouveauté, ce qu'il faut savoir apprécier c'est la combinaison harmonieuse du dialogue philosophique et des pointes satiriques de l'Ancienne Comédie contre la philosophie, ce qui est une allusion aux Nuées d'Aristophane. En revanche, il me semble plus hasardeux de voir dans les parabases del'Ancienne Comédie un modèle général des prolaliai au prétexte que, dans les deux cas, un auteur se met en scène pour se justifier et répondre aux critiques. Il s'agit là, à mon sens, d'une ressemblance due à une situation comparable, qui n'implique pas une imitation consciente des parabases.

Après l'étude de Lucien, Anna Peterson examine quelques passages des lettres d'Alciphron. Bien que l'essentiel de l'inspiration de cet auteur vienne de la Nouvelle Comédie, on peut trouver quelques allusions à l'Ancienne Comédie. C'est le cas en particulier de la lettre 2.II, où un paysan écrit à son fils d'éviter la compagnie des philosophes en faisant allusion aux Nuées d'Aristophane. Comme le fait observer justement l'autrice, nous avons affaire à une inversion et, pour ainsi dire, une normalisation: alors que chez Aristophane c'est un fils qui tente de dissuader son père de se mettre à l'école de Socrate, chez Alciphron, selon le modèle fourni par la Nouvelle Comédie, c'est un père qui s'efforce de ramener son fils dans le droit chemin. Il me semble cependant très douteux de faire intervenir les Nuées à propos de la lettre 4.7 où une hétaïre reproche à son amant de la négliger pour la compagnie des philosophes. Entre autres considérations, l’hétaïre fait valoir : «Peutêtre te semblons-nous inférieures aux sophistes parce que nous ignorons d'où viennent les nuées et à quoi ressemblent les atomes ». Il me semble évident qu'il y a ici en fait une allusion à des théories physiques dont on trouve encore un écho chez Épicure (Diogène Laërce, X, 99-Ioo).

Le dernier chapitre envisage divers auteurs du rv ${ }^{\mathrm{e}}$ s. ap. J.-C.: Thémistios, Himérios, l'empereur Julien et surtout Libanios. Au début de son autobiographie (§ 9), Libanios évoque un épisode de sa jeunesse : alors qu'il étudiait les Acharniens avec son maître, la foudre, tombant à côté d'eux, lui causa une commotion à laquelle il attribue les maux de tête dont il souffrit toute sa vie. Au lieu de voir simplement dans ce souvenir un témoignage significatif sur la place que conservait Aristophane dans la paideia des lettrés de l'Antiquité tardive, l'autrice s'efforce de montrer quel'Ancienne Comédie marque de son empreinte l'œuvre de Libanios. Pour ce faire, elle convoque surtout le témoignage d'Eunape de Sardes qui, dans ses Vies des philosophes et des sophistes, voit une influence de l'Ancienne Comédie sur le style de Libanios. Or cette analyse est extravagante: elle énumère à la fois le "charme " (charis), la "finesse» (kompsotês), la " grossièreté » (bômolochia) et “l'agrément vulgaire» (to kata thyran terpnon). Ces deux derniers traits, minimisés par l'autrice, conviennent à l'Ancienne Comédie mais sont aberrants pour Libanios, dont le style par ailleurs s'éloigne de la simplicité attique de Lucien 
ou d'Alciphron et annonce la complexité de la prose savante byzantine.

Enfin un épilogue donne un aperçu de la réception d'Aristophane à la Renaissance et plus précisément à propos de l'Utopie de Thomas More. En fait le temps d'une vraie réception d'Aristophane n'est pas encore venu lors de la publication de l'Utopie en i5ı6. À cette date, Lucien est en vogue mais pour Aristophane il faut attendre la traduction en latin des deux pièces publiées en i538. Ce décalage, ajouterais-je pour ma part, est dû au fait qu'Aristophane est un auteur moins accessible que Lucien, aussi bien pour les humanistes $d u x{ }^{e}$ s. que pour nous aujourd'hui, comme il était déjà sans doute difficile pour le jeune Libanios. Et ce n'est qu'au bout de longues années d'études que les virtuoses de la sophistique impériale étaient en mesure d'orner leurs discours de citations qui échappaient à la plupart de leurs auditeurs.

Alain Ballabriga

CNRS

a.ballabriga@gmail.com 